\title{
Ije The Journey et la néo-oralité igbo à Nollywood
}

Ije The Journey and Neo-Orality in Nollywood

\section{Françoise Ugochukwu}

\section{(2) OpenEdition}

\section{Journals}

\section{Édition électronique}

URL : https://journals.openedition.org/clo/4831

DOI : $10.4000 /$ clo.4831

ISSN : 2266-1816

Éditeur

INALCO

\section{Édition imprimée}

Date de publication : 21 juin 2018

Pagination : 127-144

ISBN : 9782858313143

ISSN : 0396-891X

\section{Référence électronique}

Françoise Ugochukwu, « lje The Journey et la néo-oralité igbo à Nollywood », Cahiers de littérature orale [En ligne], 83 | 2018, mis en ligne le 20 juin 2019, consulté le 30 juin 2021. URL : http:// journals.openedition.org/clo/4831; DOI : https://doi.org/10.4000/clo.4831

\section{(ब) (1) \&}

Cahiers de littérature orale est mis à disposition selon les termes de la Licence Creative Commons Attribution - Pas d'Utilisation Commerciale 4.0 International. 


\title{
Ije The Journey et la néo-oralité igbo à Nollywood
}

\author{
Françoise UGOCHUKWU \\ Development Policy \& Practice, Open University (GB) \& \\ CNRS-LLACAN
}

Si beaucoup de choses ont changé en vingt-trois ans dans la technique des films nigérians (coproductions internationales, usage accru des gros plans et de la voix off, plus grande qualité de son et travail post-filmique) comme dans leur contenu et leur façon d'aborder les sujets traités (avec, en particulier, une plus grande place donnée aux trajectoires individuelles et aux espaces diasporiques), ces films, décrits comme edutainment - éducation + divertissement [education + entertainment] restent avant tout porteurs d'un message, adressé, non pas seulement à l'individu mais au groupe : avatars des veillées de contes et des festivals traditionnels, ils sont destinés à être visionnés en groupe - en famille à la maison, ou dans des lieux publics tels que cafés ou salles - et à générer une discussion au sein de l'audience (Ugochukwu, 2013).

Le message des films fait d'eux les héritiers de la riche tradition orale du pays et de son rôle moral, évoquant le passé récent ou plus ancien, dénonçant les maux de leur société et guidant la réflexion du public. Cet héritage oral des films nigérians n'a pas encore été vraiment étudié : il sera observé ici dans le film Ije The Journey (2010), un film en $35 \mathrm{~mm}$ sur le thème de la violence domestique et du viol, tourné à Jos et Los Angeles. 


\section{Ije The Journey (2010)}

Ije (2010), dont le titre, igbo, accompagné de sa traduction anglaise, The Journey [le voyage], annonce la trajectoire, fait partie des films nigérians récents présentant le côté sombre du rêve américain. Ignorant les supplications de sa cadette Chioma et les conseils de son père, opposés à ses rêves de carrière et à sa poursuite du mirage américain, Anyanwu décide de partir pour Los Angeles pour se lancer dans la chanson, tandis que sa sœur restée au Nigeria devient banquière. Dix ans après, Anya, qui a épousé un Américain blanc, riche producteur de disques, est accusée d'avoir assassiné son mari et les deux inconnus qu'il avait introduits dans leur propriété de Hollywood Hills. En cellule dans l'attente du procès, elle reçoit la visite de sa sœur mais refuse de lui dire toute la vérité sur la soirée du meurtre, craignant la réaction de son père. Chioma finit par apprendre ce qui s'est réellement passé grâce aux révélations d'une codétenue, Lebe. Epaulée par Jalen Turner, le jeune avocat métis qui a accepté de défendre Anya, elle réussit alors à persuader celle-ci de tout révéler. C'est au cours du procès que sera finalement évoqué le passé traumatique des deux sœurs et de leur famille, qui explique qu'Anya ait refusé de parler jusque-là. Son témoignage devant la cour l'évocation de son enfance et du raid dont a été victime sa famille, le récit de son mariage malheureux avec un impresario adonné aux drogues et aux jeux d'argent, et celui de la soirée où il l'a violée en compagnie de ses deux acolytes - aboutit à son acquittement. Le film se termine sur les projets d'avenir du trio.

Bien que ce film porte les marques du «nouveau » Nollywood - localisation en diaspora, préférence pour les trajectoires individuelles et gros plans notamment -, on y retrouve les caractéristiques des productions de Nollywood, issues de la tradition orale :

. La centralité de la parole et de la gestuelle ;

. une structure binaire accordant une grande place au dialogue et alternant/ combinant discours et musique (chant créé pour le film et brodant sur le thème traité), présent et passé en flashbacks évoquant ici des moments particuliers de l'enfance des personnages, racontés verbalement ou en images et le plus souvent accompagnés de chants;

- une alternance de décors intérieurs (hôtels/milieu carcéral/villa des Michino/tribunal) et extérieurs (cours/chemins/champs), avec une préférence pour les espaces publics, reflétant une vie passée majoritairement en plein air et favorisant le dialogue et l'échange ; 
- un but didactique : celui d'enseigner la bonne conduite aux jeunes en mettant à profit le langage cinématographique (audiovisuel). Ce but est mis en évidence par l'épilogue traditionnel de ces films, semblable à la leçon finale des contes et qui met en scène la destruction des mauvais ou leur délivrance et/ou la récompense des bons (avec, dans les films plus anciens, une expression de remerciement comme « Gloire à Dieu !»).

\section{L'oralité comme un fil d'Ariane}

Le scénario d'Ije The Journey, opposant la trajectoire des deux sœurs, peut être considéré comme un avatar du conte-type 311 où la sœur cadette sauve son aînée des griffes de l'ogre ${ }^{1}$. Comme le révèlent les flashbacks, le film s'inscrit en outre dans le cadre des récits oraux décrits par Bano ${ }^{2}$ : celui d' « un cercle qui s'esquisse au cours de la narration : il se desserre au commencement du conte et il se ferme à sa fin ».

Le film s'ouvre (00'13-00'46) sur une prairie où deux fillettes jouent gaiement à l'oga, jeu combinant danse, battements de mains et chant, tout en devisant en igbo. Ce flashback sera suivi de quatre autres. Chioma, partie récupérer le collier dans la chambre de la villa, le trouve et se souvient : ce second retour au passé (15'56-16'58) montre les deux fillettes se querellant au sujet du collier de perles d'ambre, cadeau de la mère à Chioma, la cadette. Sa sœur, jalouse, lui arrache son collier et le jette dans le ruisseau avant de la défier d'aller le chercher. Chioma n'osant pas se risquer dans l'eau, c'est finalement Anya qui repêche le collier et le rend à sa sœur.

Le troisième flashback (24'58-26'05), qui se situe juste après l'échange (24'03-24’49) entre Anya et Lebe, une autre prisonnière, née aux États-Unis mais dont la mère est igbo, ramène le spectateur à la scène centrale de la récolte, dominée par la figure du père. À son arrivée aux champs, ce dernier trouve ses filles en train de jouer, et se met en devoir de leur montrer comment déterrer les taros. C'est l'occasion, pour Anyanwu, de protester contre l'avenir de cultivatrice qui lui est offert, d'affirmer son désir d'indépendance et de réitérer son souhait de devenir chanteuse, provoquant la colère de son père qui l'avertit de ne pas persister dans cette voie.

C'est la complainte d'une prisonnière sud-américaine qui déclenche la quatrième évocation du passé, vision du partage d'un conte populaire lors d'un

1. Aarne-Thompson, 1973, p. 101-102.

2. BANO, 1989, p. 587. 
jeu de lune traditionnel (01:02'26-01:03'31). C'est l'occasion de voir Anya diriger le chant du groupe, offrant les solos auxquels répond le groupe enfantin. L'irruption du père sur l'aire de jeu termine abruptement ce moment de bonheur : il ramène sa fille à la maison et la bat pour lui avoir désobéi cependant que Chioma, son collier d'ambre autour du cou, fait semblant de dormir. Cette évocation rassemble pour une fois les deux sœurs, qui passent cette nuit-là chacune dans sa cellule. Si Chioma ressent de la nostalgie à l'évocation du chant de sa sœur, Anya s'en souvient comme du soir où son père l'a battue et lui a encore redit son opposition à ses rêves de carrière, et elle pleure amèrement elle confiera plus tard à Chioma que l'avertissement paternel n'a jamais cessé de retentir en elle.

Le dernier flashback (01:08'40-01:10'42), le plus dramatique, amène le spectateur à revivre avec les deux sœurs le jour où leur vie insouciante a pris fin. On voit les deux fillettes assises dans un pré surplombant le village, Anya en train de coiffer Chioma tout en lui disant un conte dont la petite chante le refrain. Ce chant amène Anya à réitérer sa détermination de devenir chanteuse ; elle dit son ambition d'aller en Amérique, et de revenir riche, avec une grosse voiture dans laquelle elle promènera sa sœur et ses parents ; elle souhaite ensuite leur construire une belle maison dans une belle ville. Soudain, elles voient des hommes armés faire irruption dans le village, et se cachent dans l'école, mais un homme arrive armé d'une machette ensanglantée. On apprendra plus tard que ce jour-là, Anya a sauvé sa petite sœur en faisant face à son agresseur tandis que Chioma s'enfuyait. Chioma relatera plus tard à Jalen comment leur père est arrivé trop tard pour empêcher le viol de sa femme, mais a sauvé sa fille de justesse alors qu'elle tenait tête, debout, à son assaillant, chantant un hymne devant lui (01:22'30-01:24'10).

Les dernières images du film ramènent le spectateur au passé ludique de Chioma et de sa sœur et à Anya conduisant le conte chanté Ulule. Ces retours au passé se situent, à l'écran, dans un temps « autre », décrit par Nicolaïsen comme « hors du cadre chronologique que nous imposons habituellement au passé pour le rendre accessible », opérant une « suspension du temps historique ${ }^{3}$ » rappelant celle des contes populaires et signalant ici la présence continue du passé dans la vie et la réflexion des personnages.

3. NiCOLAÏSEN, 1989, p. 418. 


\section{La voix de la conteuse}

Le début du film met en scène la jeune femme dont le récit oral, à la première personne et adressé à sa sœur Chioma, reprend la tradition du conte, mettant en valeur l'usage de la langue, le ton, la gestuelle et la leçon morale à tirer du récit. Le reste de la projection renforcera et mettra en lumière ces différents aspects, en même temps que l'importance de la parole dans ce récit dramatique. C'est d'abord une voix off que l'on entend, presque monocorde, sur un fond chaotique où se bousculent des images violentes se succédant sans souci de chronologie et qui établissent dès le début un parallèle entre les expériences des deux sœurs. Le ton est celui du récit, intime, adressé à la sœur d'Anya, et qui suggère dès les premières paroles un secret à garder : «Ne dis surtout pas à papa ce que je vais te raconter ! »

Ce qui suit n’a pourtant rien, à première vue, qui puisse déstabiliser les relations entre le père et la fille : « Mon mari est mort, et les deux autres aussi. » Le reste du récit, volontairement décousu, ne fait qu'écorcher la surface : «Ils ne s'attendaient pas à ce que je me défende si violemment. Mais la police pense que c'est moi la coupable. Je le leur ai déjà dit : celui qui avait le pistolet n'a pas vu le verre que je tenais avant de le sentir se briser sur son cou. Il a laissé tomber le pistolet et je suis tombée à genoux pour l'attraper. »

Plus loin, Anya répète la même demande : «S'il te plaît, ne dis rien de mes problèmes à papa ! Il avait raison! » La communication verbale reste réticente, limitée, comme le montrent les interrogatoires successifs que subit Anya. Sa sœur, son avocat, la police, cherchent tous à comprendre ce qui s'est vraiment passé, mais les paroles d'Anya ne révèlent que des demi-vérités. Le silence, le non-dit, eux, sont d'autres chemins vers la révélation, du fait que « ce dont on fait silence est souvent plus présent que ce qui est explicitement exposé ${ }^{4} \gg$. C'est ce qu'illustre le récit de la soirée : on y voit Anya se débattre aux mains de ses deux assaillants, puis le mari approcher. La caméra saute ensuite brusquement hors de la villa, le récit s'arrête et seule une détonation signale que quelque chose se passe, avant que la voix d'Anya ne s'exclame : «Et je suis vivante ! II faudra attendre la fin du film pour apprendre ce qu'Anya voulait à tout prix cacher.

C'est surtout l'usage fréquent de la voix off qui caractérise ce film : elle n'est pas seulement une autoréflexion sur un événement, elle est un monologue que nous découvrons ensuite être la lecture à haute voix que fait Anya de la lettre écrite à sa sœur depuis sa cellule. Ce monologue peut aussi être reçu comme

4. BORNAND \& LEGUY, 2013, p. 165. 
un message parallèle, une histoire alternative qui appréhende la réalité derrière l'événement et surtout la lecture culturelle de cette réalité. Il est entrecoupé de silences lourds de sens et projeté sur un arrière-plan d'images parallèles (arrivée aux USA/scène du crime/arrivée au centre de détention) présentées en rapide succession et qui là encore prolongent le discours et le contextualisent.

À ces voix s'ajoute celle du père absent mais continuellement présent dans l'esprit d'Anya qui, dit-elle, l'entend tous les soirs - cette voix qui l'avertissait de ne pas s'obstiner à devenir chanteuse. Anya, après avoir raconté le viol en groupe dont elle a été victime, évoque devant les jurés l'autre viol : celui de sa mère, son assaillant et la figure protectrice de son père : « Mon père a tué l'homme avant qu'il ne puisse mettre la main sur moi. Mais les maris ne sont pas des pères. » (01:34'09-01:34'16)

\section{La structure du récit}

On retrouve dans ce film la structure du conte populaire décrite par Propp 5 , commençant par une situation - ici deux fillettes vivant une enfance insouciante au nord du Nigeria - caractérisée par un manque - ici le désir d'Anya d'embrasser la carrière de chanteuse - qui détermine son départ de la maison. On reconnaît dans la suite du film les différents moments du conte-type : interdiction du père concernant le désir de sa fille, transgression et départ pour l'Amérique, transformation du mari en agresseur et préjudice, apparition du donateur - ici la codétenue igbo - et réparation finale du méfait.

Le film est en outre construit sur une interaction constante, un dialogue entre présent et passé, Amérique et Nigeria, nuit et jour, puissamment évoqué par Anya lorsqu'elle parle des cauchemars qui hantent ses nuits et ajoute que son réveil en cellule ne fait que poursuivre le même cauchemar. Ce dialogue ne se limite pas au décor : une succession de dialogues filmés en gros plan met en valeur la profondeur de l'échange entre personnes; les sentiments et émotions retenus ajoutent du poids aux mots et prolongent le discours (une technique nouvelle à Nollywood). La succession des dialogues fait progresser le récit et l'éclaire peu à peu. C'est un dialogue d'images qui signale d'abord la profondeur de l'échange et la similarité des expériences vécues par les deux sœurs : du gros plan d'Anya rédigeant la lettre à sa sœur, on passe à celui de Chioma lisant cette même lettre. De Chioma à l'aéroport à Anya au pénitencier, les gros plans se succèdent, « moyens de dire sans dire, de recourir à l'implicite pour laisser entendre plus 
que ce que l'expression directe [...] ne peut permettre de révéler ${ }^{6} \gg$. Ces gros plans rapides sont étrangement similaires : contrôles et photos d'identité en miroir, et fouilles répétées. Ce même dialogue entre passé et présent est évoqué par les photos posées côte à côte dans la chambre des Michino : un gros plan de deux visages heureux, puis deux gros plans de visages mornes, séparés et se tournant le dos. Anya commentera pendant le procès : « J'ai joué le jeu comme une bonne épouse, jusqu'à ce... qu'il devienne quelque chose d'autre. »

Comme l'explique une jeune codétenue, « Anya ne vous a pas tout dit. [...] Elle pense protéger un secret, mais ici, on n'est pas en Afrique. Ça aidera si le jury est au courant. Quelle est la seule chose qu'Anya ne voudrait absolument pas que votre père apprenne, même si cela l'amène à risquer sa liberté ? » (01:07’39-01:07’42) Tentant plus tard d'expliquer à l'avocat cette « culture de la honte $\gg$, Chioma se souvient alors (01:08'40-01:10'42) des émeutes du nord, de l'irruption des Hausa dans le village et du viol de sa mère, rejetée ensuite par leur père comme « un bien endommagé ». Cet épisode est suivi d'une longue discussion entre Chioma et Jalen sur le viol, Chioma expliquant le stigmate qui accompagne le viol au Nigeria, et les efforts de sa sœur pour garder le silence sur ce qui s'est passé le soir du crime. Ce n'est qu'à la fin, au tribunal, que nous apprendrons, grâce au témoignage d'Anya, comment, violée par son mari qui s'était joint aux deux visiteurs, Anya n'a réussi à se libérer qu'en blessant mortellement ses assaillants. Au fur et à mesure, le dialogue des voix et des images lève le voile sur le secret d'Anya, qui, découvert, perd son pouvoir empoisonné. Comme le dira Anya à sa sœur : « Tu m’as rendu ma vie ! »

\section{La gestuelle et le regard}

En l'absence d'un récit complet, ce sont les gros plans sur les visages qui donnent la clé du récit, « délivrent des informations supplémentaires, apportent des nuances expressives, complètent les informations, ajoutent des éléments nouveaux, rendent compte du caractère des personnages et de leurs émotions ${ }^{7}$ » et offrent au spectateur un chemin vers la vérité. Là encore, le film adapte à l'écran la notion de performance si présente dans le contage traditionnel, utilisant au mieux cet outil qui, selon le réalisateur et cinéaste français Jean Renoir (1894-1979), « différencie le cinéma de toutes les autres formes de spectacle ». Dans le même entretien télévisé, Renoir commentait l'impact du

6. BORNAND \& LEGUY, 2013, p. 168.

7. BAUMGARDT, 2008, p. 70. 
gros plan sur les spectateurs : «C'est brusquement un lien mystérieux et très fort entre ce personnage qui est sur l'écran et les gens dans la salle [qui] pénètrent dans cet acteur, dans cette actrice [...], s'identifient à lui ou à elle. » Il concluait en qualifiant l'effet d' « hypnotique ${ }^{8} \gg$.

Allongée sur son lit, Anya parle à sa sœur : « Je voudrais tant revoir ton visage ! » Comme en réponse apparaît un gros plan du visage de Chioma. Le film est fait de nombreux gros plans, qui permettent d'observer et de lire les visages : le visage baigné de larmes d'Anya par exemple, tout au début, qui dit son désarroi ; son genou bandé ; le corps de son mari, avachi sur sa chaise, les cuisses écartées avec la tache de sang au niveau du cœur ; Chioma lisant la lettre de sa sœur dans l'avion. Les sourires sont rares et coïncident presque toujours avec l'évocation du passé et du bonheur familial. Selon Geneviève Calame-Griaule, qui a contribué à relever dans son œuvre « le rôle essentiel de la gestuelle dans la narration »:

Les gestes favorisent la compréhension du texte ; ils peuvent aussi remplacer certaines expressions trop fortes, et surtout ils captivent le public en «dramatisant » (au sens étymologique de $\ll$ mise en action $»)$ le récit ${ }^{9}$.

Dans le film, certains gestes sont particulièrement symboliques : celui de Chioma pénétrant dans sa chambre d'hôtel et allumant la lumière (elle arrive en Amérique pour faire la lumière sur la situation qui a amené sa sœur en prison); celui d'Anya, la main sur la gâchette du pistolet de son mari face à ses assaillants. D'autres gestes sont « liés profondément à la culture ${ }^{10} \gg:$ c'est ici le cas de ceux qui introduisent le spectateur à la culture des deux sœurs et rappellent à quel point elles restent étrangères à leur environnement américain ; celui de Chioma élevant la coupelle et la faisant tourner au-dessus de la tête d'Anya, simulant le rite de deuil des veuves; le ntoo d'Anya à sa sœur, les deux mains tirant le haut des pommettes vers le bas, expression igbo enfantine de moquerie pouvant se traduire par « ça te va bien! »

\title{
La musique
}

La musique joue un rôle important dans les films de Nollywood. Toujours présente, elle accompagne le récit, le commente et en facilite le décryptage. Le

\author{
8. RENOIR, 1971. \\ 9. Calame-Griaule, 2008, p. 93.
}

10. BAUMgardT, 2008, p. 69. 
chant, genre hérité de l'oralité, s'y trouve renouvelé : désormais œuvre d'auteur et créé spécialement pour chaque film, il remplit d'abord une fonction artistique, ajoutant à la beauté du film et le replaçant dans une culture particulière. Il commente aussi l'action, décode le non-dit et/ou offre un moment de réflexion entre deux moments d'action, réflexion facilitée par ce ralentissement du déroulement du film. Ce film est tout entier enveloppé de musique : la mélopée sur laquelle s'ouvre le récit rappelle que Chioma vit à Lagos, en territoire yoruba. Le chant igbo qui sert de fond sonore à la dernière scène dit l'identité retrouvée.

La musique est d'abord et clairement à la source du récit - elle représentait le rêve d'Anya, comme nous l'apprend la scène du champ de taros au cours de laquelle la pré-adolescente exprime son souhait de devenir chanteuse et tient tête à l'opposition catégorique de son père. Elle devient un peu plus tard l'arme avec laquelle la fillette paralyse l'action du violeur en chantant un hymne, sauvant ainsi la vie à sa petite sœur. Elle est enfin au cœur de la vie professionnelle de la jeune chanteuse en Amérique. C'est son amour de la musique qui a amené Anya là où elle est. Pendant le flashback sur la scène du triple crime, la caméra, après s'être portée sur les corps sans vie des trois hommes, se fixe sur l'écran de télévision silencieux qui continue à projeter le visage d'Anya en concert (01:35'01-01:35'23). Cette brève image annonce à la fois la victoire de la jeune femme et son avenir : le viol n'a pas tué le chant en elle, et va lui permettre, non seulement de survivre mais de réussir sa vie.

La musique commente l'action à sa manière. La mélopée yoruba qui accompagne l'arrivée de Chioma à Los Angeles rappelle Lagos, la mégapole qu'elle a quittée pour venir voir sa sœur ; elle la marque aussi comme nigériane. Plus tard, c'est la complainte de la prisonnière sud-américaine qui ramène Chioma, en cellule pour une nuit après avoir été surprise en train de détruire des comprimés trouvés chez sa sœur, dans le passé heureux des jeux de lune et au chant des contes que dirigeait sa sœur. Pour elle, le chant et le destin de sa sœur sont intimement liés, et la mélodie résume ce qu'il y a de meilleur dans Anya : la beauté, le talent. Ce même chant, Anya l'entend, mais, s'il la ramène elle aussi dans le passé, c'est pour lui rappeler que son père l'avait frappée cette nuit-là pour être sortie chanter : dans sa mémoire, le chant devient ainsi le symbole de l'interdit, de la désobéissance, du chemin qu'elle a choisi contre l'avis de son père et de l'abîme où l'a plongée la recherche du succès. Le chant a pour elle un goût amer.

Chioma, vers la fin du film, donne à sa sœur la possibilité de rejeter cette perception négative de son talent, de tourner la page, de revenir à la source de son rêve et de retrouver la vision qu'elle en avait autrefois. Abordant pour la première fois, au cours d'une conversation-clé, le secret de sa sœur et sa peur du 
rejet de leur père, Chioma révèle à Anya ce que ce dernier lui a récemment appris, évoquant le jour de l'attaque de leur village : comment « l'homme à la machette restait là, figé, pendant que tu chantais un hymne, le défiant de t'attaquer pendant que ton Dieu écoutait. Tu n'as pas cessé de chanter, même après que papa l'a égorgé. Anya, lève-toi et affronte ta peur demain, comme tu l'as fait pour moi ce jour-là ! » (01:23’30-01:24'10)

\section{Les objets-aides}

On sait qu' « une place particulière est à accorder aux petits objets de conte, aux objets matériels. On sait l'importance qu'ils ont au sein d'une œuvre et comment ils servent de ponctuation symbolique dans le déroulement du processus créateur $^{11} \gg$. Dans ce film, les objets, en gros plan, jouent un rôle essentiel : comme ceux dont se sert la conteuse, comme ceux aussi qui apparaissent à la charnière des grands moments du conte, ces objets magiques mis à la disposition $\mathrm{du}$ héros ${ }^{12}$, ils sont là pour focaliser l'attention de l'auditoire et faire avancer le récit. Ils ont aussi un pouvoir symbolique et renforcent le lien entre les deux sœurs.

- La feuille de mauvais papier sur laquelle Anya a écrit à sa sœur depuis sa cellule est le premier objet sur lequel se concentre l'attention : on voit d'abord Anya en train d'écrire, au crayon, allongée sur son lit. La feuille réapparait ensuite dans les mains de Chioma dans l'avion qui l'amène à Los Angeles. Cette feuille permet de représenter concrètement la pensée du personnage principal ; elle sert aussi de moyen mnémotechnique, fixant visuellement le sujet du film.

- La coupelle en bois, contenant un liquide rougeâtre dans lequel surnagent des brins d'herbe, symbolise le rite funéraire auquel doivent encore se soumettre les veuves, au Nigeria, avant les funérailles de leur mari. D'abord présentée comme le prétexte qui permet à Chioma d'accéder à sa sœur en détention, elle fait le lien avec la famille restée au pays et avec sa culture qui rend ses racines à la jeune femme exilée, même si les deux sœurs s'entendent pour rejeter ce rite humiliant. Elle sert enfin de paravent aux deux sœurs et facilite leur échange en détournant l'attention de la gardienne.

11. Chouvier \& Morhain, 2010, p. 164.

12. Propp, 1970, p. 55. 
. Le collier de perles d'ambre, qui figure dès le titre du film, soulignant le mot Ije, est clairement le plus important, et peut être considéré comme un objet-proverbe, « symbolisant un énoncé [et] substitué à la parole ${ }^{13}$ » qui résume ici la totalité du récit. Mentionné par Anya dans sa lettre à sa sœur, il a une longue histoire qui nous est contée peu à peu. Chioma, la cadette, l'avait reçu en cadeau de leur mère et Anya, qui avait longtemps jalousé sa sœur pour ce précieux souvenir, l'a subtilisé avant de partir en Amérique. Maintenant incarcérée, elle confesse ce chapardage à sa sœur, exprime le regret que la magie du collier n'ait pas opéré et demande à Chioma d'aller le récupérer chez elle avant que la famille de son mari ne le prenne. Ce collier, symbole du lien entre les deux sœurs, successivement distendu, brisé et réparé, les relie aussi à leur mère perdue et à la culture de leur pays d'origine. Dans la dernière scène, sur la plage, Anya rend le collier à Chioma pour lui porter chance au moment où celle-ci se prépare à suivre Jalen chez lui, retrouvant les gestes de leur ancienne querelle pour les défaire : elle jette le collier, non plus dans l'eau mais sur le sable, où sa cadette, maintenant adulte et libérée de ses peurs anciennes, le récupère. Il convient de noter que ce collier est le seul objet à propos duquel est fait mention d'un pouvoir magique. Anya lui avait clairement attribué les vertus d'un talisman. Comme le rappelle Xavier Garnier, « la défaillance de l'objet magique est expliquée par le mauvais comportement du héros ${ }^{14}$ », ici la transgression de l'interdiction du père par Anya. Porté à nouveau par Anya lors de son témoignage à la fin de son propre procès, le collier d'ambre retrouvre son pouvoir magique à mesure que la honteuse vérité sort de la bouche de la jeune femme que son courage sauvera.

D’autres objets représentent d'autres liens, éphémères ceux-là :

. Le pistolet de Michael, qui ne le quitte jamais, tombera finalement dans les mains d'Anya qui s'en servira pour se libérer de ses assaillants.

. La médaille de la Vierge, trouvée sur le sol de la maison par Chioma et qui se révèle appartenir à Caroline, la femme de ménage des voisins, permet de renouer le lien entre Caroline et Anya et de décrypter ce qu'Anya cherchait à cacher.

13. BAUMGARDT, 2008, p. 71.

14. GARNIER, 2001, p. 478. 


\section{La langue du film}

Comme le soulignait Praline Gay-Para :

Le conteur doit rendre l'émotion que porte le conte, embarquer son auditoire dans son voyage. Cela n'est possible que si le sens des mots est immédiatement accessible [...]. Le problème se pose avec plus d'acuité pour les conteurs qui transmettent leur culture d'origine, différente de celle d'ici. Dans un premier temps, la langue constitue une difficulté. Comment faire passer en français des termes, des expressions, des manières de dire propres à la langue d'origine ${ }^{15}$ ?

Le film peut être considéré comme la traduction du conte en langage cinématographique, technique qui consiste à dérouler le conte « comme un scénario..., pour essayer de [...] faire sentir à quel point c'est de l'image que naît le conte, c'est de l'image que naît la parole du conte », le conteur devenant alors « un montreur d'images ${ }^{16}$ ». Tout au long du film, la langue des échanges et la prononciation même de l'anglais par les différents personnages balisent la compréhension et mettent en avant le lien entre langue, identité et projection de soi dans un espace étranger. Les deux langues employées par les personnages disent leur identité en même temps qu'elles les placent géographiquement et culturellement. Les scènes du passé présentent le père aux champs avec ses filles et l'igbo y est naturellement le seul moyen de communication. Plus tard, les deux sœurs vont vivre hors de leur zone linguistique - Chioma à Lagos, ville cosmopolite où l'anglais domine la vie quotidienne et son milieu professionnel, et Anya aux États-Unis. Si la première rencontre des deux sœurs après dix ans d'absence se fait en anglais, langue qui symbolise ici une relation distendue, elles reviendront rapidement à l'igbo, langue clairement présentée tout au long de leur interaction comme la langue de l'intimité, celle du bonheur et de l'harmonie, même si leurs conversations restent souvent caractérisées par l'alternance codique.

Le nom donné aux personnages des contes ne confirme pas seulement leur identité ethnique : il annonce leur parcours et permet de deviner le dénouement. Dans Ije, les deux sœurs portent des prénoms igbo - Chioma [bonne destinée] et Anyanwu [soleil], dont le récit confirmera la validité : Chioma, qui a « réussi »

15. Gay-PARA, 1999, p. 120.

16. BLOCH, 1999, p. 170. 
puisqu'elle a un bon métier, sauvera la vie à sa sœur comme Anya lui avait sauvé la vie des années auparavant. Anya, elle, s'est très tôt distinguée par sa voix et a réussi à percer en tant que chanteuse en Amérique. Plusieurs scènes - à l'hôtel, à la douane, en prison - soulignent en revanche les difficultés liées à l'annonce du nom (Chioma/Anyanwu Opara) et la défiance qu'il génère d'emblée, même si Anyanwu offre un diminutif facilitant sa prononciation - le sourire d'Anya en entendant la policière prononcer son nom, et son commentaire : « Anya... c'est nigérian », dit sa fierté igbo. On pourrait ajouter que le nom prononcé la ramène au Nigeria en pensée.

Le chant, partie intégrante des films, accompagne et commente l'action, brode sur le thème principal et prolonge le script. Il a également un rôle de remueur d'émotions auprès du public. Ces caractéristiques expliquent et justifient les langues du chant - les langues nigérianes, même si un certain nombre de films nigérians récents ont fait le choix de l'anglais dans le but de toucher un public diasporique. Tout au long d'Ije par exemple, la mélopée dans une langue nigériane - yoruba au début du film, igbo dans la dernière scène qui accompagne la voix off en arrière-plan dit le mal du pays d'une exilée qui se raccroche au passé et à sa culture comme à une bouée. Le film exprime en outre une autre facette, rarement mise en avant, du caractère igbo (et nigérian) : la fierté de sa langue et de son identité, ravivée encore par l'immersion en milieu étranger.

Le langage des films ne se limite pas à la parole. Le support audiovisuel offre, on l'a vu, une riche palette et emprunte son langage à la culture dominante du film : coupe, couleurs et texture des vêtements, gestuelle, regards et mimiques (amplifiés dans les films plus récents par l'usage du gros plan), occupation de l'espace et instruments de musique traditionnels (flûtes, tambours) sont mis à contribution pour enrichir le discours. Dans Ije, l'évocation du passé au Nord du Nigeria se fait par exemple entièrement au travers de jeux traditionnels (oga) et de conversations en igbo, marquant d'autant plus l'identité des deux jeunes femmes que celle-ci s'est développée hors de leur zone linguistique. L'importance de ce langage cinématographique est confirmé par Abecera selon lequel la translation du récit oral au cinéma « va plus loin que faire passer l'anecdote [...] à l'image » et $\ll$ suppose une réécriture absolue ${ }^{17} \gg$. 


\section{La leçon morale}

Ije The Journey, bien qu'appartenant à la catégorie du nouveau Nollywood, modèle somme toute assez récent, respecte jusqu'au bout la structure traditionnelle de ces films, en particulier leur cadre éducatif et leur message moral. Le film tout entier est destiné à l'éducation et à la transmission d'informations culturelles : les images ne se contentent pas d'évoquer, elles informent sur une histoire nationale mouvementée et ses conflits inter-ethniques, sur les cultures et coutumes du Nigeria, sur la langue igbo, en même temps que sur les raisons de la migration et les relations raciales aux États-Unis. Ces leçons sont nombreuses, adressées à la police et au système judiciaire, encouragés à plus d'objectivité, et à la société tout entière invitée à ne pas juger sur l'apparence et à montrer plus de sympathie pour la cause des femmes. Le film offre aussi une réflexion sur les dangers de l'émigration, visant à une conscientisation du public.

Confrontée à une autre culture, Anya s'efforce d'informer sur le Nigeria. Ce film peut en outre être considéré comme ayant exploré la notion d'edutainment plus loin que les autres en soutenant ouvertement, avant même le générique, la Fondation du Vou ${ }^{18}$ - une partie de l'argent des ventes du film est destinée à soutenir la campagne de la Fondation contre les violences domestiques affectant les femmes.

La réflexion sur le thème brûlant de l'émigration, illustrée par l'arrivée aux États-Unis, première destination des Nigérians, au sein d'une queue composée de Blancs, met d'abord en avant le racisme des douaniers qui traitent Chioma différemment du reste des voyageurs. Le procès d'Anya révèlera ensuite et peu à peu le caractère composite du pays d'accueil - Américains blancs, latinos, asiatiques et métis - et une ségrégation faite d'ignorance et de préjugés, la difficulté de se faire comprendre et la violence des relations qui en découle. Le dialogue révèle les divergences entre Anya et Chioma à ce propos : si la seconde dit avoir espéré le retour de sa sœur et continue à penser qu'elle n'aurait jamais dû s'expatrier, pour Anya et en dépit de tout, «il n'y avait rien pour [elle] » au Nigeria - elle considérait l'Amérique, et non la richesse au Nigeria, comme « la porte du ciel ». Jetée derrière les barreaux pour avoir tenté de faire disparaître les comprimés susceptibles d'incriminer sa sœur, Chioma aura cette réflexion désabusée : «On dit que l'Amérique est la porte du ciel. Combien ont déjà vu l'autre côté ?»

18. www.takeavow.org 
Comme le rappelait G. Calame-Griaule,

l'enseignement traditionnel utilise la littérature orale pour donner aux jeunes des leçons de comportement [...]. Plus profondément, le conte s'adresse à l'inconscient et pose, sous une forme symbolique, les problèmes de relations humaines qui préoccupent toutes les sociétés [...]. Dans les sociétés modernes, le conte est en train de retrouver cette fonction pédagogique millénaire ${ }^{19}$.

Nollywood, le nouveau cinéma nigérian, présent aujourd'hui dans toute l'Afrique et au-delà, a pris le relais du conte populaire pour proposer une forme d'enseignement ludique à de nouveaux auditoires et inspirer de nouvelles formes d'art.

\section{Sources}

Aarne Antti \& Stith Thompson, 1999, The Types of the Folktale, $2^{\mathrm{e}}$ édition, Suomalainen tiedeakatemia/Academia Scientarum Fennica, Helsinki, 588 p.

Abecera Richard, 1999, « Le medium du conte, c'est la parole » in Calame-Griaule Geneviève (dir.), Le Renouveau du conte, Éditions du CNRS, Paris, p. 174-178.

BANO Istvan, 1984, « L'analyse esthétique et la composition des contes populaires » in Calame-Griaule Geneviève \& GÖRÖG-KARADY Veronika (dir.), Le conte, pourquoi ? comment? - Actes des journées d'études en littérature orale, analyse des contes, problème de méthodes, Éditions du CNRS, Paris, p. 585-594.

BAUMgardT Ursula, 2008, « La performance » in BAUMgardT Ursula \& DERIVE Jean (dir.), Littératures orales africaines : perspectives théoriques et méthodologiques, Karthala, Paris, p. 49-75.

BAUmgaRdT Ursula \& DeRIVE Jean (dir.), 2008, Littératures orales africaines : perspectives théoriques et méthodologiques, Karthala, Paris, 439 p.

19. Calame-Griaule, 1999, p. 16. 
Bloch Muriel, 1999, « Le conteur et son film conducteur » in Calame-Griaule Geneviève (dir.), Le Renouveau du conte, Éditions du CNRS, Paris, p. 168-170.

Bornand Sandra \& Leguy Cécile, 2013, Anthropologie des pratiques langagières, Armand Colin (coll. Collection U. Sciences humaines \& sociales), Paris, 205 p.

Calame-Griaule Geneviève, 1975, « Nouvelles recherches sur le langage gestuel des conteurs » in Journal des Africanistes, $\mathrm{n}^{\circ}$ 45-1, p. 198, DOI : 10.3406/jafr.1975.2193.

Calame-Griaule Geneviève (dir.), 1999, Le renouveau du conte, Éditions du CNRS, Paris, $449 \mathrm{p}$.

Calame-Griaule Geneviève, 2008, « Dites-le avec des gestes. Comment étudier la gestuelle des conteurs ? » in Cabiers de littérature orale, $n^{\circ}$ 63-64, p. $83-108$, DOI : $10.4000 /$ clo.106.

Calame-Griaule Geneviève \& Görög-Karady Veronika (dir.), 1984, Le conte, pourquoi ? comment ? - Actes des journées d'études en littérature orale, analyse des contes, problème de méthodes, Éditions du CNRS, Paris, 628 p.

Chineze Anyaene (réal.), 2010, Ije The Journey, 109 minutes.

Chouvier Bernard \& Morhain Yves, 2010, « Le conte. Une parole virtuelle qui s'actualise $\gg$ in Cabiers de psychologie clinique, $\mathrm{n}^{\circ} 2$, vol. 35, p. 163, DOI : $10.3917 /$ cpc.035.0163.

Garnier Xavier, 2001, « Remarque sur le contrôle de l'efficience magique dans le conte des objets magiques $\gg$ in Diop Papa Samba \& LÜSEBRINK Hans-Jürgen (dir.), Littératures et sociétés africaines : regards comparatistes et perspectives interculturelles : mélanges offerts à János Riesz à l'occasion de son soixantième anniversaire, G. Narr, Tübingen.

GaY-Para Praline, 1999, « Le répertoire du conteur » in Calame-Griaule Geneviève (dir.), Le Renouveau du conte, Éditions du CNRS, Paris, p. 115-122. 
NiCOLAÏSEN Wilhem T.H., 1984, "The structure of narrated time in the folktale" in Calame-Griaule Geneviève \& Görög-Karady Veronika (dir.), Le conte, pourquoi ? comment? - Actes des journées d'études en littérature orale, analyse des contes, problème de méthodes, Éditions du CNRS, Paris, p. 417-436.

Propp Vladimir, 1970, Morphologie du conte suivi de Les Transformations des contes merveilleux et de l'Étude structurale et typologique du conte, Seuil, Paris, $254 \mathrm{p}$.

ReNOIR Jean, 1971, Entretien, INA, www.ina.fr/video/105124029.

Ugochukwu Françoise, 2013, Nollywood on the Move: Nigeria on Display, Wissenschaftlicher Verlag Trier, Trier, $260 \mathrm{p}$.

Résumé : la plupart des critiques s'accordent sur le fait que la sortie du film igbo Living in Bondage (1992) marque le début du cinéma populaire nigérian, qui s'est construit en reprenant les fonctions des récits oraux, érigeant les personnages-clés en conteurs modernes, et laissant le plus souvent une liberté quasi-totale à l'improvisation à partir d'un canevas didactique bâti autour de l'événement à présenter. Cette production est concurrencée depuis quelques années par des films plus spécialement destinés à la projection en salle, qui, tout en conservant le fondement oral traditionnel, contribuent à ériger les bases d'un nouveau cinéma. Cet héritage oral des films nigérians n'a pas encore été vraiment étudié : il sera observé ici dans le film Ije The Journey (2010), un film en $35 \mathrm{~mm}$ sur l'envers du rêve américain, tourné à Jos et Los Angeles.

Mots-clefs : Nigeria, Igbo, littérature orale, néo-oralité, cinéma, Nollywood, migration.

\section{Ije The Journey and Neo-Orality in Nollywood}

Abstract: most critics agree that the release of the Igbo film Living in Bondage (1992) marks the beginning of the Nigerian popular cinema, which took over the functions of oral narratives, turning key characters into modern storytellers and allowing actors a quasi-total freedom of improvisation, using a didactic outline as a guide. This production has been challenged in recent years by films primarily intended for big screens, which, while retaining their traditional oral model, 
belp building the foundations of a new cinema. This oral heritage of Nigerian films has not yet been really studied: it will be considered here in the film Ije The Journey (2010), a $35 \mathrm{~mm}$ film on the dark side of the American dream, set in Jos and Los Angeles.

Keywords: Nigeria, Igbo, oral literature, neo-oralite, cinema, Nollywood, migration.

\section{Note sur l'auteur}

Françoise Ugochukwu est spécialiste de littérature comparée, d'études africaines et d'ethnolinguistique. En poste au Royaume-Uni après plus de vingt ans à l'université de Nsukka au Nigeria, elle est l'auteure de plusieurs ouvrages et de nombreuses publications sur les littératures africaines orales et écrites, la langue et la culture igbo du Nigeria et le cinéma nigérian. 